\title{
Non-Convex Penalized Estimation of Count Data Responses via Generalized Linear Model (GLM)
}

\author{
Rasaki Olawale Olanrew ajuํㅜ and Johnson Funminiyi Ojo ${ }^{2}$ \\ ${ }^{1}$ University of Ibadan \\ Department of Statistics (Ibadan, Nigeria) \\ Email: rasakiolawale [AT] gmail.com \\ ${ }^{2}$ University of Ibadan \\ Department of Statistics (Ibadan, Nigeria) \\ Email: jfunminiojo [AT] yahoo.co.uk
}

\begin{abstract}
This study provided a non-convex penalized estimation procedure via Smoothed Clipp ed Absolute Deviation (SCAD) and Minimax Concave Penalty (MCP)for count data responses to checkmate the problem of covariates $(d)$ exceeding the sample size $(n)$. The Generalized Linear Model (GLM) approach was adopted in obtaining the penalized functions needed by the MCPand SCAD non-convex penalizations of Binomial, Poisson and Negative-Binomial related count responses regression. A case study of the colorectalcancer with six (6) co variates against sample size offive (5) was subjected to the non-convexpenalized estimation of the three distributions. It was revealed that the non-convex penalization of Binomial regression via MCP and SCAD best explained four unpenalized covariates needed in determining whether surgical or therapyidealfor treating the turmoil.
\end{abstract}

Keywords - Count Data, MinimaxConcave Penalty (MCP), Non-convexpenalization, Smoothed Clipped Absolute Deviation (SCAD).

\section{INTRODUCTION}

Linear regression analysis of high-throughput and high dimensional datain bioinformatics, neurosciences, clinical studies etc. often impede with the number of covariates $(d)$ exceeding the number of the sample size $(n)[14,17]$. Efficient methods of variable selection via shrinking of covariate(s) and sparse estimation of reg ression coefficients techniques have been propounded based on penalized likelihood function (loss function) and regularizing parameter" $\eta "$. Among the techniques proposed to circumvent aforesaid challenge is the folded concave penalty function of Least Absolute Shrinkage and Selection Operator (LASSO) proposed by [11] and either by Smooth ed Clipped Absolute Deviation (SCAD) or minimax concave loss function of Minimax Concave Penalty (MCP) as proponed by [3] and [15] respectively.

Unlike the convexpenalty, where the likelihood functions of LASSO, SCAD and MCP influence bias edness in their parameter estimations via absolute values, non-convex(non-concave) penalty of MCP and SCAD relieves the absolute value constrain in concave LASSO in order to eliminate the biasedness influence [18,6]. Non-conv ex penalty satisfies a wide range of statistical properties; ability not to only fix finite estimates of regression coefficients $\left(\beta_{i}\right)$ but also to estimate true zero regression coefficients with their probabilities approximately equals one to confirm their sufficiency. In addition, the ability of non-convex MCP and SCAD to estimate non-zero coefficients as if the true sparsely is known [10].

In this paper, the Probability Mass Functions (PMFs) of count data responses (dependent variables) of Binomial, Poiss on and Negative-Binomial distributions will be assumed to follow a linear regression responses when $d>n$. The non-convexpenalized estimations of these count linear regression responses will be subdued to loss (penalized) functions of SCAD and MCP via Generalized Linear Model (GLM). Moreover, the solution of the penalized regression coefficients will be via proximal coordinate iterative procedure because of its tractable global solution faster rate of convergencefor a pre-selected regularized parameter" $\eta$ " and high dimensional selection criteria. 


\section{SPECIFICATION OF THE NON-CONVEX PENALIZED ESTIMATION VIA GLM}

Given independent randomvariables and a set of covariates $\left(y_{i}, x_{i}\right) i=1, \ldots \ldots, n$ for a randomsample from a linear regression

$$
Y=X \beta+\varepsilon
$$

Where $Y=\left(y_{1}, y_{2}, \ldots, y_{n}\right)^{T}$ is the response vector matrix, $X$ is the $n$ by $d$ designmatrix, $\beta=\left(\beta_{0}, \beta_{1}, \ldots, \beta_{n}\right)^{T}$ is the vectorof regression coefficients while $\varepsilon=\left(\varepsilon_{1}, \varepsilon_{2}, \ldots, \varepsilon_{n}\right)^{T}$ is the vector matrix of the random component of a Generalized Linear Models (GLM) such that its Probability Density Function PDF or Probability Mass Function(PMF) belongs to the exponential family;

$$
f(y ; x, \beta)=f\left(y_{i} ; \theta_{i}, \phi\right)=c\left(y ; \theta_{i}\right) \exp \left[\frac{y_{i}\left(\theta_{i}\right)-b\left(\theta_{i}\right)}{a(\phi)}\right]
$$

Where $\left(\theta_{1}, \theta_{2}, \ldots, \theta_{n}\right)^{T}=X \beta=b\left(\theta_{i}\right)$ is the natural or canonical parameter, $y_{i}\left(\theta_{i}\right)$ is the canonical form, $a(\phi)$ is the scale parameter for $\phi \in(0, \infty), c\left(y ; \theta_{i}\right)$ is the function with " $y$ " only.

[3], [7] and [8] maintained that the approach for estimating regression coefficients of a high dimensional data via non-convex regularization or penalization to be

$$
\begin{aligned}
& Q(\beta)=\operatorname{Min}_{\beta \in \Gamma^{d}}\left\{\frac{1}{n} L(\beta)+f_{\eta}(\beta)\right\} \\
& =\operatorname{Min}_{\beta \in \Gamma^{d}}\left\{\frac{1}{n}(X \beta-y)^{2}+f_{\eta}(\beta)\right\}
\end{aligned}
$$

Where $L(\beta)$ is the loss function (the log function of equation (2)), $f_{\eta}(\beta)$ is the non-con vex penalized or regularized function of either for SCAD or for MCP with turning parameter(otherwise known as regu larized parameter " $\eta$ " that must satisfy the standard optimization solution of $\beta_{\eta}$ for a first-order Karus h-KuhnTucker (KKT) condition of

$$
h=\partial\left\{L\left(\beta_{\eta}\right)+f_{\eta}\left(\beta_{\eta}\right)\right\}
$$

[3], [11] and [17] defined non-convexpenalty for SCAD as

$$
f_{\eta}\left(\beta_{k}\right)= \begin{cases}\eta\left|\beta_{k}\right| & \text { if } \beta_{k} \leq \eta \\ \frac{a \eta\left|\beta_{k}\right|-\frac{1}{2}\left(\beta_{k}^{2}+\eta^{2}\right)}{a-1} & \text { if } \eta<\left|\beta_{k}\right| \leq a \eta \\ \frac{\eta^{2}(a-1)}{2(a-1)} & \text { if } \beta_{k}>\eta\end{cases}
$$

For " $a$ " which is a fixed parameter $>2 ; \eta>0$

Also, [6], [15] and [16] claimed that the non-convexpenalty for MCP to be

$$
f_{\eta}\left(\beta_{k}\right)= \begin{cases}\eta\left(\left|\beta_{k}\right|-\frac{\beta_{k}^{2}}{2 \eta b}\right) & \left|\beta_{k}\right| \leq \eta b \\ \frac{\eta^{2} b}{2} & \left|\beta_{k}\right|>\eta b\end{cases}
$$


$" b "$ Which is a fixed parameter $>0$

$$
\text { Otherwise, } f_{\eta}\left(\beta_{k}\right)= \begin{cases}\frac{\operatorname{sgn}\left(\beta_{k}\right)\left(\left|\beta_{k}\right|-\eta\right)_{+}}{1-b} & \text { if }\left|\beta_{k}\right| \leq \eta b \\ \beta_{k} & \text { if }\left|\beta_{k}\right|>\eta b\end{cases}
$$

The non-convexpenalty $f_{\eta}\left(\beta_{k}\right)$ can be decomposed into sumof penalty and sumof the concave part;

$$
f_{\eta}(\beta)=\sum_{k=1}^{d} f_{\eta}\left(\beta_{k}\right)=\eta\|\beta\|+\sum_{k=1}^{d} g_{\eta}\left(\beta_{k}\right)
$$

This could be simplified by rewriting as

$$
G_{\eta}(\beta)=\sum_{k=1}^{g} g_{\eta}\left(\beta_{k}\right)=f_{\eta}(\beta)-\eta\|\beta\|
$$

$G_{\eta}(\beta)$ connotes the dis integration of concave part of the non-convexpenalty $f_{\eta}(\beta)$.

[1], [9], [12] and [13] as serted that the regularized solution for the regression coefficients could be compressed to a coordinate proximal method via Newpton-Raphson method iteration for update via

$$
\varphi_{L_{m}^{c}}, \eta_{m}\left(\beta, \beta_{m}^{c+1}\right)=\beta_{m}^{c-1}-\frac{1}{L_{m}^{c}} H_{\eta}
$$

such that, $H_{\eta}=\nabla L\left(\beta_{\eta}\right)+\nabla G_{\eta}\left(\beta_{\eta}\right)$ for a chosen " $\eta$ " and surrogate $L_{m}^{c}>0$ formof $L_{\eta_{m}}, \beta_{m}^{c}$ corresponds to the $c^{\text {th }}$ iteration within $t^{\text {th }}$ path solution and " $N$ " the number of path stages.

$$
N=\frac{\log \left(\frac{\eta_{0}}{\eta_{n d}}\right)}{\log \left(\gamma^{-1}\right)}
$$

$\eta_{n d}$ scales of sample size " $n "$ and the dimension $" d "$ and chosen $\gamma$, and via the selection of turning parameter (regularized parameter) " $\eta "$ by model selecon criteria of either[2], [4], [5], or [14].

$$
\begin{gathered}
A I C=-2 L(\beta)+2 d f(\eta), B I C=-2 L(\beta)+\log (N) d f(\eta) \\
C A I C=-2 L(\beta)+\log (N+1) d f(\eta) \\
E B I C_{\eta}(s)=-2 \log L(\beta)+v_{(s)} \log (N)+2 \gamma \log \left(\begin{array}{l}
d \\
k
\end{array}\right)
\end{gathered}
$$

Where " $d f "$ is the degree of freedomof non-zero parameters. $0 \leq \gamma<1 s \subset\{1, \ldots . ., d\}, \theta_{(s)}$ is the parameter $\beta$ whose components outside " $s$ " being set to be zero or some pre-selected values, $\beta_{(s)}$ is the maximum likelihood estimator of $\beta_{(s)}$ while $v_{(s)}$ is the number of component(s). 


\section{PENALIZED ESTIMATION VIA MCP AND SCAD}

\subsubsection{Binomial Penalized Regression Estimation}

$$
\begin{gathered}
\text { Assume } Y_{i} \square \operatorname{Bin}\left(n_{i}, p_{i}\right) \text {. Then the PMF; } P\left(p_{i} ; y_{i}, n_{i}\right)=\left(\begin{array}{c}
n_{i} \\
y_{i}
\end{array}\right) p_{i}{ }^{y_{i}}\left(1-p_{i}\right)^{n_{i}-y_{i}} y_{i}=1, \ldots \ldots, n_{i} \\
=\exp \left\{y_{i} \log \frac{p_{i}}{1-p_{i}}+n_{i} \log \left(1-p_{i}\right)-\log \left(\begin{array}{c}
n_{i} \\
y_{i}
\end{array}\right)\right\} \quad \text { (10) } \\
\qquad \begin{array}{c}
b\left(\theta_{i}\right)=n_{i}\left(1+\exp \left(\theta_{i}\right)\right), \theta_{i}=\log \frac{p_{i}}{1-p_{i}}, c\left(y_{i} ; \theta_{i}\right)=-\log \left(\begin{array}{c}
n_{i} \\
y_{i}
\end{array}\right), \phi=1, \\
a(\phi)=1, E\left(Y_{i}\right)=n_{i} p_{i}, V\left(Y_{i}\right)=n_{i} p_{i}\left(1-p_{i}\right)
\end{array}
\end{gathered}
$$

Where $p_{i}$ is the probability of success and $1-p_{i}$ is the probability of failure.

\subsubsection{SCAD Non-Convex Penalized Binomial Regression}

From equation(10), the link function of Binomial $\mu(X \beta)=p_{i}=\frac{\exp \left(\beta^{\prime} x_{i}\right)}{1+\exp \left(\beta^{\prime} x_{i}\right)}$ called Logit function

$$
\begin{gathered}
Q_{\eta}(\beta)=-\frac{1}{n} \sum_{i=1}^{n}\left\{\left(y_{i} \beta^{\prime} x_{i}\right)-\log \left(1+\exp \left(\beta^{\prime} x_{i}\right)\right)\right\}+G_{\eta}(\beta) \\
f_{\eta(S C A D)}\left(\beta_{k}\right)= \begin{cases}\frac{-\beta_{k}^{2}-2 a \eta\left|\beta_{k}\right|+\eta^{2}}{2(a-1)} & \text { for }\left|\beta_{k}\right| \leq \eta \\
\frac{(a+1) \eta^{2}}{2} & \text { for } \eta<\left|\beta_{k}\right| \leq a \eta\end{cases} \\
g_{\eta S C A D}\left(\beta_{k}\right)= \begin{cases}\frac{2 \eta\left|\beta_{k}\right|-\beta_{k}^{2}}{2(a-1)} & \text { for }\left|\beta_{k}\right|>a \eta \\
\frac{(a+1) \eta^{2}-2 \eta\left|\beta_{k}\right|}{2} & \text { for }\left|\beta_{k}\right|>a \eta\end{cases}
\end{gathered}
$$

With non-convexloss function

$$
\begin{aligned}
& \nabla_{B I N} L(\beta)=\frac{1}{n} \sum_{i=1}^{n} x_{i}\left\{\frac{\exp \left(\beta^{\prime} x_{i}\right)}{1+\exp \left(\beta^{\prime} x_{i}\right)}-y_{i}\right\}, \\
& \nabla G_{\eta B i n}(\beta)_{S C A D}=\left\{\begin{array}{ll}
\eta_{m} \operatorname{sgn}\left(\beta_{k}\right)-\beta_{k} & \text { for } \eta_{m}<\left|\beta_{k}\right| \leq a \eta_{m} \\
-\eta_{m} \operatorname{sgn}\left(\beta_{k}\right) & \text { for } \quad\left|\beta_{k}\right|>a \eta_{m}
\end{array} \quad \text { For } a>2\right.
\end{aligned}
$$

Updating for optimals solution

$$
\beta_{m(S C A D)}^{c+1}=\beta_{m}^{c-1}-\frac{1}{L_{m}^{c}}\left\{\nabla G_{\eta B i n}\left(\beta^{c-1}\right)_{S C A D}+\nabla_{B I N} L\left(\beta^{c-1}\right)\right\} V_{B I N}{ }^{c-1}
$$

Where $V_{B I N}=\operatorname{diag}\left\{p_{1}^{c}\left(1-p_{1}^{c}\right), \ldots \ldots, p_{n}^{c}\left(1-p_{n}^{c}\right)\right\}$

Until convergent of the vector of regres sion coefficients is reached in equation (12) with starting value $\beta_{m}^{c} \neq 0$ and $\mathrm{N}^{\text {th }}$ number of path stages. 


\subsubsection{MCP Non-Convex Penalized Binomial Regression}

From equation(11),

$$
\begin{aligned}
& f_{\eta M C P}\left(\beta_{k}\right)= \begin{cases}\left(\eta\left|\beta_{k}\right|-\frac{\beta_{k}^{2}}{2 b}\right) & \text { for }\left|\beta_{k}\right| \leq b \eta \\
\frac{b \eta^{2}}{2} & \text { for }\left|\beta_{k}\right|>b \eta\end{cases}
\end{aligned}
$$

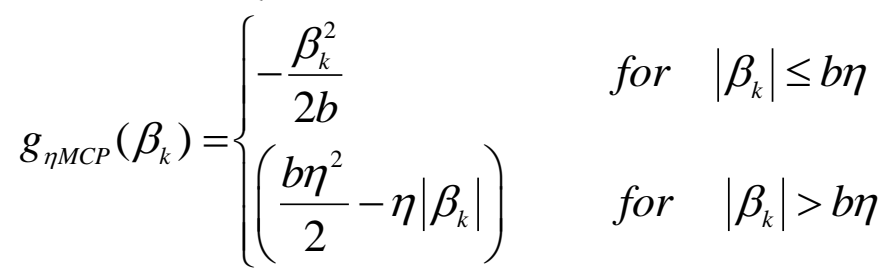

$$
\begin{aligned}
& \nabla_{M C P} G_{\eta}\left(\beta_{k}\right)= \begin{cases}-\frac{\beta_{k}}{b} \eta_{m} \operatorname{sign}\left(\beta_{k}\right) & \text { for }\left|\beta_{k}\right| \leq b \eta_{m} \\
-\eta_{m} \operatorname{sign}\left(\beta_{k}\right) & \text { for }\left|\beta_{k}\right|>b \eta_{m}\end{cases}
\end{aligned}
$$

For $b>0$, updating for convergence,

$$
\beta_{m(M C P)}^{c+1}=\beta_{m}^{c-1}-\frac{1}{L_{m}^{c}}\left\{\nabla_{M C P} G_{\eta B i n}\left(\beta^{c-1}\right)+\nabla L\left(\beta^{c-1}\right)\right\} V^{c-1}
$$

Until convergent of the vector of regres sion coefficients is reached in equ ation (13) with starting value $\beta_{m}^{c} \neq 0$ and $\mathrm{N}^{\text {th }}$ number of path stages.

\subsection{Poisson Penalized Regression Estimation}

Assume $Y_{i} \square \operatorname{Poisson}\left(\lambda_{i}\right)$. Then the PMF;

$$
\begin{gathered}
P\left(\lambda_{i} ; y_{i}\right)=\exp \left(\lambda_{i}\right) \frac{\lambda_{i}^{y_{i}}}{y_{i} !} y_{i}=0,1, \ldots \ldots \\
=\exp \left\{y_{i} \log \left(\lambda_{i}\right)-\lambda_{i}-\log \left(y_{i} !\right)\right\} \\
\theta_{i}=\log \left(\lambda_{i}\right), b\left(\theta_{i}\right)=\exp \left(\theta_{i}\right), \phi=1, a(\phi)=1, c\left(y_{i} ; \theta_{i}\right)=-\log \left(y_{i} !\right) \\
E\left(Y_{i}\right)=V\left(Y_{i}\right)=\lambda_{i}
\end{gathered}
$$

Where $\lambda_{i}$ is the parameter of interest

The link function of Poisson $\mu\left(X^{\prime} \beta\right)=\exp \left(\beta^{\prime} x_{i}\right)=\log \left(\lambda_{i}\right)$ called Probit function. Then the criterion for the SCAD penalized Pois son regression

$$
\begin{gathered}
Q_{\eta}(\beta)=\frac{1}{n} \sum_{i=1}^{n}\left\{\exp \left(\beta^{\prime} x_{i}\right)-\left(y_{i} \beta^{\prime} x_{i}\right)\right\}+G_{\eta}(\beta) \\
g_{\eta S C A D}\left(\beta_{k}\right)=\left\{\begin{array}{lc}
\frac{2 \eta\left|\beta_{k}\right|-\beta_{k}^{2}}{2(a-1)} & \text { for } \quad \eta<\left|\beta_{k}\right|<a \eta \\
\frac{(a+1) \eta^{2}-2 \eta\left|\beta_{k}\right|}{2} & \text { for } \quad\left|\beta_{k}\right|>a \eta
\end{array}\right. \\
\nabla_{P O I} L(\beta)=\frac{1}{n} \sum_{i=1}^{n} x_{i}\left\{\exp \left(\beta^{\prime} x_{i}\right)-y_{i}\right\}
\end{gathered}
$$




$$
\nabla G_{\eta P o i}(\beta)_{S C A D}=\left\{\begin{array}{lc}
\eta_{m} \operatorname{sgn}\left(\beta_{k}\right)-\beta_{k} & \text { for } \eta_{m}<\left|\beta_{k}\right| \leq a \eta_{m} \\
-\eta_{m} \operatorname{sgn}\left(\beta_{k}\right) & \text { for } \quad\left|\beta_{k}\right|>a \eta_{m}
\end{array}\right.
$$

Updating for convergence, $\beta_{m(S C A D)}^{c+1}=\beta_{m}^{c-1}-\frac{1}{L_{m}^{c}}\left\{\frac{1}{n} \sum_{i=1}^{n} x_{i}\left\{\exp \left(\beta^{c-1} x_{i}\right)-y_{i}\right\}+\nabla G_{\eta P O I}\left(\beta^{c-1}\right)_{S C A D}\right\} V_{P O I}^{c-1}$

Where, $V_{P O I}=\operatorname{diag}\left\{\exp \left(\beta^{c} x_{1}\right), \ldots . ., \exp \left(\beta^{c} x_{n}\right)\right\}$

\subsubsection{MCP Non-Convex Penalized Poisson Regression}

Fromequation(14)

$$
\begin{aligned}
& g_{\eta M C P}\left(\beta_{k}\right)=\left\{\begin{array}{lll}
-\frac{\beta_{k}^{2}}{2 b} & \text { for } & \left|\beta_{k}\right| \leq b \eta \\
\left(\frac{b \eta^{2}}{2}-\eta\left|\beta_{k}\right|\right) & \text { for } & \left|\beta_{k}\right|>b \eta
\end{array} \text { For } b>0\right. \\
& \nabla_{P O I} L(\beta)=\frac{1}{n} \sum_{i=1}^{n} x_{i}\left\{\exp \left(\beta^{\prime} x_{i}\right)-y_{i}\right\} \\
& \nabla_{M C P} G_{\eta P O I}\left(\beta_{k}\right)= \begin{cases}-\frac{\beta_{k}}{b} \eta_{m} \operatorname{sign}\left(\beta_{k}\right) & \text { for }\left|\beta_{k}\right| \leq b \eta_{m} \\
-\eta_{m} \operatorname{sign}\left(\beta_{k}\right) & \text { for }\left|\beta_{k}\right|>b \eta_{m}\end{cases}
\end{aligned}
$$

For $b>0$

$$
\beta_{m(M C P)}^{c+1}=\beta_{m}^{c-1}-\frac{1}{L_{m}^{c}}\left\{\frac{1}{n} \sum_{i=1}^{n} x_{i}\left\{\exp \left(\beta^{c-1} x_{i}\right)-y_{i}\right\}+\nabla_{M C P} G_{\eta P O I}\left(\beta^{c-1}\right)\right\} V_{P O I}^{c-1}
$$

until convergence of the vector of regres sion coefficients is reached in equation (16) with starting value $\beta_{m}^{c} \neq 0$ and $\mathrm{N}^{\text {th }}$ number of path stages.

\subsection{Negative-Binomial Penalized Regression Estimation}

Assume $Y_{i} \square N B\left(k, p_{i}\right)$.Then the PMF;

$$
\begin{gathered}
P\left(r, p_{i} ; y_{i}\right)=\left(\begin{array}{c}
y-1 \\
r-1
\end{array}\right) p_{i}^{r}\left(1-p_{i}\right)^{y_{i}} y=r, r+1, r+2 \ldots . . \\
=\exp \left\{y_{i} \log \left(1-p_{i}\right)+r \log \frac{p_{i}}{1-p_{i}}-\log \left(\begin{array}{c}
y-1 \\
r-1
\end{array}\right)\right\} \\
b(\theta)=-r \log \frac{1-\exp (\theta)}{\exp (\theta)}, c\left(y_{i} ; \theta_{i}\right)=-\log \left(\begin{array}{c}
y-1 \\
r-1
\end{array}\right), \theta=\log \left(1-p_{i}\right), a(\phi)=1, \\
E\left(Y_{i}\right)=\frac{r}{p}=\frac{r}{1-\exp (\theta)}, V\left(Y_{i}\right)=\frac{r(1-p)}{p^{2}}=\frac{r \exp (\theta)}{(1-\exp (\theta))^{2}}
\end{gathered}
$$

$" r "$ Is the shape parameter for meas uring the deg ree of clumping or ag gregation (dispersion). 


\subsubsection{SCAD Non-Convex Penalized Negative-Binomial Regression}

The link function of Negative Binomial $\mu\left(X^{\prime} \beta\right)=\eta=\frac{1}{1+\exp \left(\beta^{\prime} x_{i}\right)}=p_{i}$ called log of the log function. Then the criterion for the SCAD penalized Neg ative-Binomial regression.

$$
\begin{aligned}
Q_{\eta}(\beta)=-\frac{1}{n} \sum_{i=1}^{n}\left\{\left(y_{i} \beta^{\prime} x_{i}\right)-\log \left\{\frac{1-\exp \left(\beta^{\prime} x_{i}\right)}{\exp \left(\beta^{\prime} x_{i}\right)}\right\}\right\}+G_{\eta}(\beta) \\
g_{\eta S C A D}\left(\beta_{k}\right)= \begin{cases}\frac{2 \eta\left|\beta_{k}\right|-\beta_{k}^{2}}{2(a-1)} & \text { for } \eta<\left|\beta_{k}\right|<a \eta \\
\frac{(a+1) \eta^{2}-2 \eta\left|\beta_{k}\right|}{2} & \text { for }\left|\beta_{k}\right|>a \eta\end{cases}
\end{aligned}
$$

Then the non-convex loss function

$$
\nabla_{N B} L(\beta)=\frac{1}{n} \sum_{i=1}^{n} x_{i}\left\{\log \left\{\frac{1}{1+\exp \left(\beta^{\prime} x_{i}\right)}\right\}-y_{i}\right\}
$$

$\nabla G_{\eta N B}(\beta)_{S C A D}=\left\{\begin{array}{ll}\eta_{m} \operatorname{sgn}\left(\beta_{k}\right)-\beta_{k} & \text { for } \eta_{m}<\left|\beta_{k}\right| \leq a \eta_{m} \\ -\eta_{m} \operatorname{sgn}\left(\beta_{k}\right) & \text { for } \quad\left|\beta_{k}\right|>a \eta_{m}\end{array}\right.$ For $a>2$

Updating foroptimal regres sion coefficient

$$
\beta_{m(S C A D)}^{c+1}=\beta_{m}^{c-1}-\frac{1}{L_{m}^{c}}\left\{\nabla_{S C A D} G_{\eta N B}\left(\beta^{c-1}\right)+\nabla L\left(\beta^{c-1}\right)\right\} V_{N B}{ }^{c-1}
$$

For $V_{N B}=\operatorname{diag}\left\{\frac{r \exp \left(\beta^{\prime c} x_{1}\right)}{\left(\exp \left(\beta^{\prime c} x_{1}\right)\right)^{2}}, \ldots ., \frac{r \exp \left(\beta^{\prime c} x_{n}\right)}{\left(\exp \left(\beta^{\prime c} x_{n}\right)\right)^{2}}\right\}$

Until convergence of the vector of regression coefficients is reached in equation(18) with starting value $\beta_{m}^{c} \neq 0$ and $\mathrm{N}^{\text {th }}$ number of path stages.

\subsubsection{MCP Non-Convex Penalized Negative-Binomial Regression}

From equation (17),

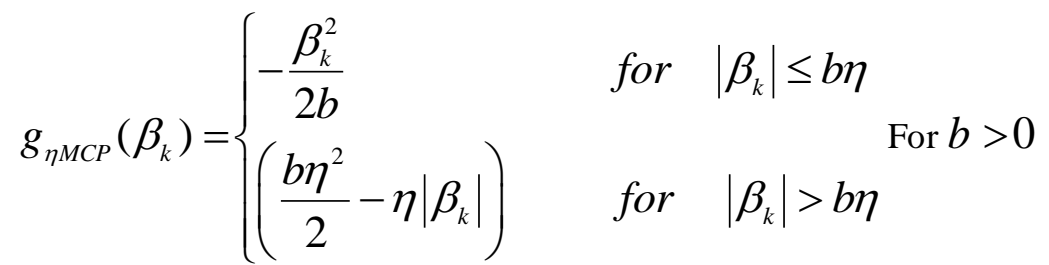

$$
\begin{aligned}
& \nabla_{N B} L(\beta)=\frac{1}{n} \sum_{i=1}^{n} x_{i}\left\{\log \left\{\frac{1}{1+\exp \left(\beta^{\prime} x_{i}\right)}\right\}-y_{i}\right\} \\
& \nabla_{M C P} G_{\eta N B}\left(\beta_{k}\right)= \begin{cases}-\frac{\beta_{k}}{b} \eta_{m} \operatorname{sign}\left(\beta_{k}\right) & \text { for }\left|\beta_{k}\right| \leq b \eta_{m} \\
-\eta_{m} \operatorname{sign}\left(\beta_{k}\right) & \text { for }\left|\beta_{k}\right|>b \eta_{m}\end{cases}
\end{aligned}
$$

Updating foroptimal regres sion coefficient

$$
\beta_{m(M C P)}^{c+1}=\beta_{m}^{c-1}-\frac{1}{L_{m}^{c}}\left\{\nabla_{M C P} G_{\eta N B}\left(\beta^{c-1}\right)+\nabla L\left(\beta^{c-1}\right)\right\} V_{N B}{ }^{c-1}
$$


Until convergence of the vector of regression coefficients is reached in equation(19) with starting value $\beta_{m}^{c} \neq 0$ and $\mathrm{N}^{\text {th }}$ number of path stages.

\section{ANALYSIS}

A study case of colorectal cancer was identified from the Nnamdi Azikwe teaching hospital, Anambra state, Nigeria from 2014 to 2017. Colorectal cancer is a pelvic swollen turmoil in male patients .The data comprises of six (6) covariates $d=6$ as against the sample size of five (5) $n=5$. The covariates are influences or factors that determined whether the the swollen pelvic will be subjected to operation or uses of drugs in order to stop/suppress the growing turmoil. The count covariates are age in years, status = level of progression, nodes=size of the turmoil (s mall or large cell can cer), e type $=$ type of the cells, $r x=$ level of completion of cancer therapy, and obstruct $=$ chronic or acute level of the unwanted growth. From the stated date, only five cases were recorded.

Table 1: Pois s on coefficients of model criteria, penalty, selected variables, res idual deviance and penalized residual.

\begin{tabular}{cccc}
\hline Measures & MCP & SCAD & GLM \\
\hline BIC & 14.4463 & 14.4463 & 20.5098 \\
\hline AIC & 15.2274 & 15.2274 & 21.2270 \\
\hline L1 (Loss function) & -11.22741 & -11.22741 & -- \\
\hline Log-likelihood & -5.6137 & -5.6137 & -5.6137 \\
\hline Deviance & 0.8345 & 0.8345 & $8.3457 \mathrm{e}-01$ \\
\hline Residual de viance & 1.7492 & 1.7492 & $2.5596 \mathrm{e}-21$ \\
\hline SelectedVariable(s) & 4 & 4 & 4 \\
\hline PV & 0.0009 & 0.0014 & -- \\
\hline PR & 2.3798 & 2.4056 & -- \\
\hline RP & 0.02449 & 0.02439 & - \\
& $\mathrm{a}=3$ & $\mathrm{~b}=3.7$ & \\
\hline
\end{tabular}

Keys: PV= Penalized Value; PR= Penalized Residual; RP= Regularized Parameter

Table 2: Binomial coefficients of model criteria, penalty, selected variables, residual deviance and penalized residual.

\begin{tabular}{clcc}
\hline Measures & MCP & SCAD & GLM \\
\hline BIC & 5.5705 & 4.5171 & 9.2030 \\
\hline AIC & 6.3516 & 3.7360 & 10.000 \\
\hline Li(Loss function) & -2.3516 & -0.5171 & ---- \\
\hline Log-likelihood & -7.1758 & -7.2586 & -7.0261 \\
\hline De viance & 0.3012 & 0.30112 & $6.7301 \mathrm{e}+00$ \\
\hline Residual deviance & 0.55167 & 0.317173 & $2.1434 \mathrm{e}-10$ \\
\hline SelectedVariable(s) & 4 & 4 & 4 \\
\hline PV & 0.0009 & 0.00141 & -- \\
\hline PR & 0.28033 & 0.16563 & -- \\
\hline RP & 0.02449 & 0.02449 & $\mathrm{~b}=3.7$ \\
\hline
\end{tabular}

Keys: PV= Penalized Value; PR= Penalized Residual; RP= Regularized Parameter 
Table 3: Negative-Binomial coefficients of model criteria, penalty, selected variables, residual deviance and penalized residual.

\begin{tabular}{cccc}
\hline Measures & MCP & SCAD & GLM \\
\hline BIC & 14.4675 & 14.4675 & 18.4901 \\
\hline AIC & 15.2486 & 15.2486 & 19.0686 \\
\hline L1 (Loss function) & -11.2486 & -11.2486 & -- \\
\hline Log-likelihood & -5.6137 & -5.6243 & -5.6243 \\
\hline Deviance & 0.8395 & 0.8394 & 0.9686 \\
\hline Residual de viance & 1.7492 & 1.7492 & 1.9631 \\
\hline Selected Variable(s) & 4 & 4 & 4 \\
\hline PV & 0.0009 & 0.00139 & -- \\
\hline PR & 2.3798 & 2.4056 & -- \\
\hline RP & 0.02439 & 0.02439 & -- \\
\hline Dispersion & 0.0252 & 0.0251 & \\
\hline
\end{tabular}

Keys: PV= Penalized Value; PR= Penalized Residual; RP= Regularized Parameter

From the three dis tributional tables, two covariates "status" and "nodes" were penalized while four covariates "extent", "age", "etype" and "rx" are the significant and contributing factors in the threedifferent distribution responses considered (selected variables). It is obvious that Binomial non-convex penalized estimation has the minimum error model selection criteria of BICs of $(5.5705,4.5171)$ and AICs of $(6.3516,3.7360)$ for MCP and SCAD respectively as against a higher BICs and AICs of $(14.4463,14.4463)(14.4675,14.4675)$ and $(15.2274,15.2274)(15.2486,15.2486)$ for Poiss on and Negative BinomialMCPs and SCADs res pectively. In collaboration with the model selectio $\mathrm{n}$ criteria, the $\log$-likelihood of the Binomial MCP and SCAD happened to besmallest of all the log-likelihoods of the distributions. This affirmed the assertion that the smaller the log-likelihood theideal and robust the model. The penalized values for the MCPs and SCADs of the probability distributions coincide but their residuals vary. The penalized errors of Bin omial function for MCP and SCAD are $(0.28033 \& 0.28033)$ respectively compared to Poisson and Negative Binomial MCPs and SCADs of same value at (2.3798 \& 2.4056). It is to be noted that the GLM approach indirectly penalized covariates by ignoring irrelevant covariates without necessary provision for penalization. 

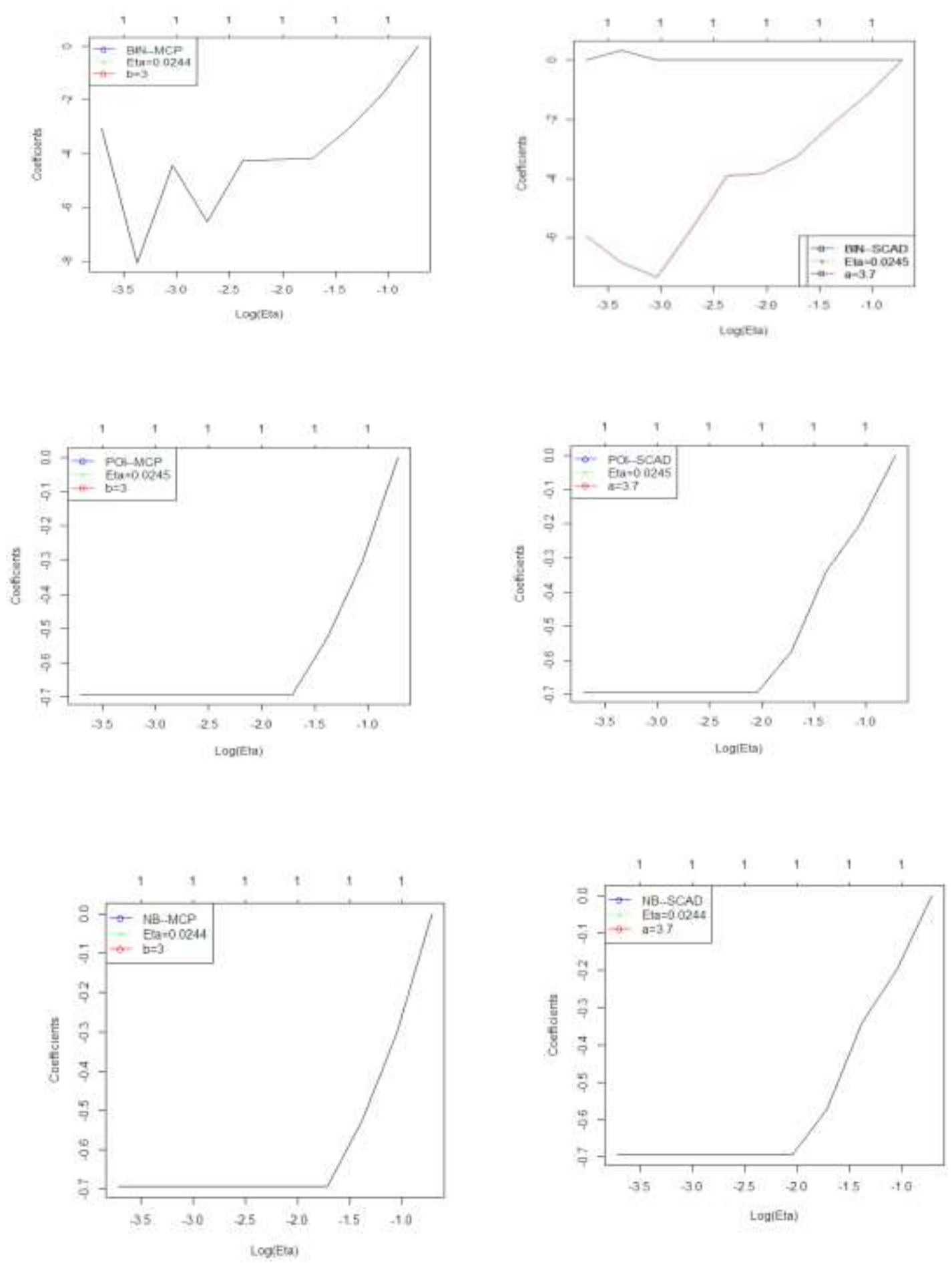

Figure 1: Coefficient Plot of the Coefficient Paths for the Fitted MCPs and SCADs.

Figure 1. shows the path fitted of the regression coefficients by each of the MCPs and SCADs of the considered probability mass distributions. Having said that each of the non-convex penalized estimation selected four cov ariates, Binomial non-convexpenalizations of estimates of $\beta s$ showed a more non-homogeneity in nature for conformity as ag ainst a parallel estimates by Poisson and Negative Binomial non-convexpenalizations. Figure 1 buttresses the aberrant of the actual responses in the MCPs, SCADs of Poisson, and Negative Binomial non-convex penalization to their predicted responses. Moreover, figure 1. elucidates on the high magnitude of values suppressed by Poisson and Negative Binomial dis tributions in the process of penalizing based on the considered case study. In addition, Neg ative Bin o mial 
dis tribution estimated a s maller value for the frequency distribution scattering froman average, th at is, the Negative Binomial responses revealed an over-dis persion of $2.5 \%$ by the covariates, which is of ne gligible consequence as to affecting the estimated parameters. Furthermore, The GLM approach was out-performed by the meas urement indexes of the non-convex of the penalizations.
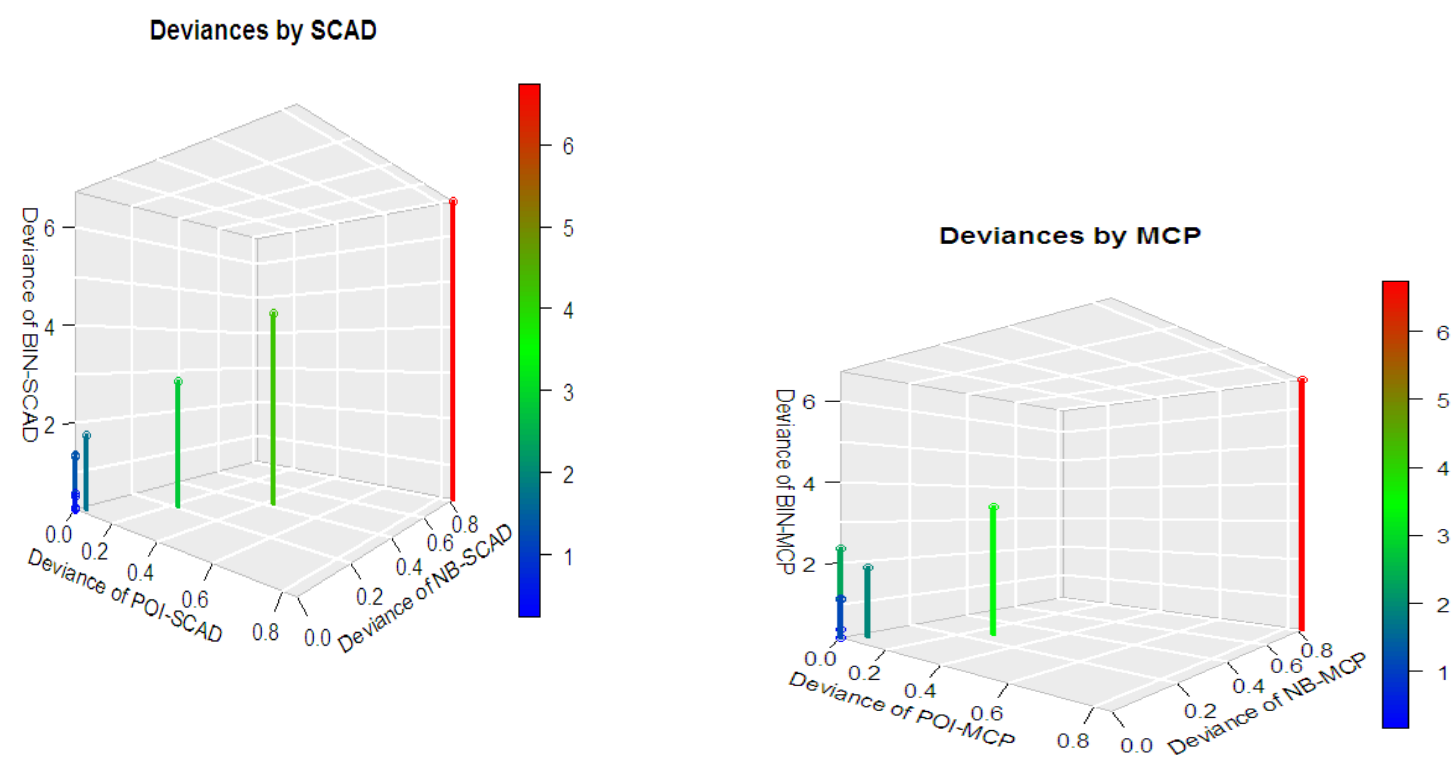

Figure 2: Deviance plot of the MCPs and

SCADs via Binomial, Pois son Negative Binomialdis tributions.

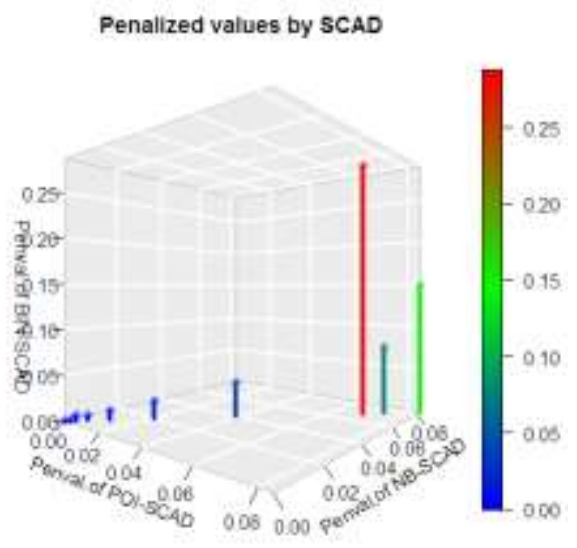

Figure 3: Penalized values by each of the MCPs and

SCADs in

Binomial, Pois son Negative Binomial dis tributions.

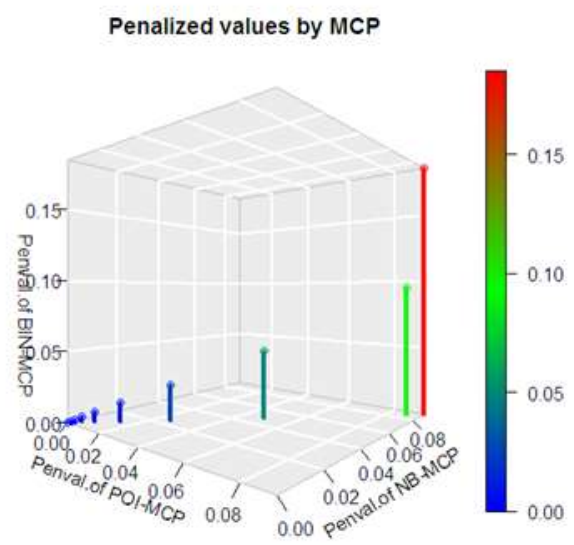

\section{CONCLUSION}

Given the previous analysis, it is safe to state that the Binomialrelated responses of non-convexpenalization via MCP and SCAD was pre-eminent in the regulation of factors contributing to a surgical or drug use approach in curbing colorectalcancervia penalization of covariates. Apart fromthe Pois son, Binomial and Negative Bin omial res ponses non-convex penalization considered, it should be noted that responses with different PMFs or PDFs could also be constrained to the non-convex penalization via GLM provided they belong to the exponential family. However, the Ordinary Least Square (OLS) could also be considered as an alternative approach for the GLM when circumventing the problemof number of covariates exceeding the sample size via MCP and SCAD. 


\section{ACKNOWLEDGMENT}

We would like to acknowledge and thank the core management of Nnamdi Azikwe teaching hospital, Anambra state, Nigeria for providing datasets on of colorectal cancer for this research.

\section{REFERENCES}

[1] Agarwal, A., Negahban, S. and Wain wright, M. J, "Fast global convergence of gradient methods for highdimensional statis tical recovery" Annals of Statis tics, vol. 40, no. 5, pp 2452-2482, 2012. doi:10.1214/12-AOS1032.

[2] Chen, J. and Chen, Z., "Extended Bayesian information criteria for model selection with large model spaces" Biometrika, vol.95, no.3, pp. 759-771, 2008.doi:10.1093/biomet/asn034.

[3] Fan, J. and Li, R., "Variable selection via non-concave penalized likelihood and its oracle properties" Journal of the American Statistical Association, vol. 96, no. 456, pp. 1348-1360, 2001. doi: 10.1198/016214501753882273.

[4] Hirose, Kand Yamamoto, M., "Penalized likelihood factor analysis via non-convexpenalties" $5^{\text {th }}$ International Conference of the ERCIM WG on Computing \& Statistics (ERCIM 2012), Conference Centre, Oviedo, Spain, pp.1-33, December 2012.

[5] Kim, Y., Kwon, S. and Choi, H, "Consistent model selection criteria on high dimensions" Journal of Machine Learning Research, vol. 13, no. 1, pp. 1037-1057, 2012.

[6] Liu, H.Y. Yao, T. and Li, R, "Global solutions to folded concave penalized non-convexlearning" The Annals of Statistics. Vol. 44, no.2, pp.629-659, 2016. doi:10.1214/15-A OS1380. (O Institute of Mathematical Statistics.

[7] Loh, P-L. and Wainwright, M. J, "Regularized M-estimators with non-convexity: Statistical and algorithmic theory for local optima" Journal of Machine Learning Research, vol. 16, pp. 559-616, 2015.

[8] Loh, P-L, "Local optima of non-convexregularized M-estimators" ElectricalEngineering and Computer Sciences University of California at Berkeley. Technical Report No. UCB/EECS-2013-54, 2013. http://www.eecs.berkeley.edu/Pubs/TechRpts/2013/EECS-2013-54.html

[9] Nesterov, Y, "Gradient methods for minimizing composite functions" Mathematical Programming, vol.140, no. 1, pp. 125-161, 2013. Doi:10.1007/s 10107-012-0629-5.

[10] Wang, Z., Han, H. and Zhang, T, “Optimal computational and statis tical rates of convergence for sparse non-convex learning problems" The Annals of Statis tics, vol. 42, no. 6, pp. 2164-2201, 2014. doi: 10.1214/14-AOS1238.

[11] Wang, L., Kim, Y. and Li, R, "Calibrating non-convexpenalized regression in ultra-high dimension" Annals of Statistics, vol.41, no. 5, pp. 2505-2536, 2013. doi: 10.1214/13-AOS1159 @ Institute of Mathematical Statistics.

[12] Xiao, L., Zhang, T., “A proximal-gradient homotopy method for the sparse least-squares problem” SIAM Journal on Optimization, vol. 23, no. 2, pp. 1062-1091, 2013.

[13] Yang, N. and Han, L., "A general theory of hypothesis tests and confidence regions for sparse high dimensional models" The Annals of Statistics, vol. 45, no. 1, pp. 158-195, 2017. doi:10.1214/16A OS1448.

[14] Yu, Y. and Feng, Y., “APPLE: approximate path for penalized likelihood estimators” Journal of Statistical Computing, vol. 24, pp. 803-819, 2014. doi: 10.1007/s 11222-013-9403-7.

[15] Zhang, C.H., "Nearly unbiased variable selection under minimax concave penalty" Annals of Statistics, vol. 38, no. 2, pp. 894-942, 2010. doi: 10.1214/09-aos729.

[16] Zhang, C.-H and Zhang, T., "A general theory of concave regularization for high dimensional sparse estimation problems" Statistical Science, vol. 27, no. 4, pp. 576-593, 2012.

[17] Zhang, K., Yin, F. and Xiong, F., "Comparis ons of penalized least squares methods by simulations” 1-16, 2014. arXiv:1405.1796v1 [stat. Co].

[18] Zhong, L.W. and Kwok, J.F., "Gradient descent with proximal average for non-convexand composite regularization, Proceedings of the Twenty-Eighth AAAI Conference on Artificial Intelligence, pp. 1- 7, 2014. 